\title{
Erratum to: Inhibition of ovarian cancer cell growth by a novel TAK1 inhibitor LYTAK1
}

\author{
Liu Wei ${ }^{1} \cdot$ Liu Ying $^{2} \cdot$ Yin Chunxia ${ }^{3}$
}

Published online: 8 October 2015

(C) Springer-Verlag Berlin Heidelberg 2015

\section{Erratum to: Cancer Chemother Pharmacol} (2015) 76:641-650

DOI 10.1007/s00280-015-2822-8

Unfortunately, the order and affiliations of authors have been wrongly published. The correct order is given below:

Liu Wei, Liu Ying and Yin Chunxia

Liu Wei and Liu Ying are co-first authors.

The online version of the original article can be found under doi:10.1007/s00280-015-2822-8.

Yin Chunxia

dryinchunxia71@yeah.net

1 Department of Radiology, Jilin City Central Hospital, Jilin,

China

2 Central Laboratory, Maternal and Child Health Center in Jilin

Province, Changchun, China

3 Obstetrics and Gynecology Hospital of Changchun City,

555 West Wuma Road, Nanguan District, Changchun

City 130042, Jilin Province, China 